\title{
AN IMPROVED ESTIMATE IN THE METHOD OF FREEZING
}

\author{
ROBERT E. VINOGRAD
}

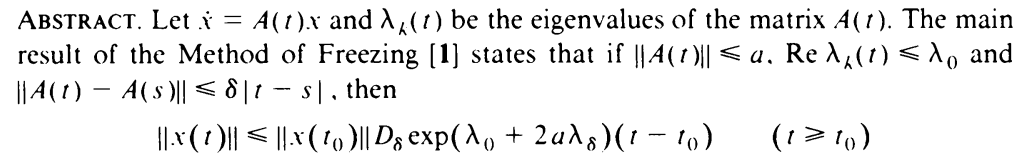

for all solutions of the system, where

$$
\lambda_{\delta}=\left(C_{n} \cdot \delta / 4 a^{2}\right)^{1 /(n+1)} .
$$

The previous best known value. $C_{n}=n(n+1) / 2$, is reduced to the substantially smaller value $2 n^{n} e^{-n} /(n-1) !<\sqrt{2 n / \pi}$.

The main result of the Method of Freezing [1] for linear differential equations can be stated as follows:

Let an $n$-dimensional system

$$
\dot{x}=A(t) x
$$

be given and let $\lambda_{k}(t)$ be the eigenvalues of the matrix $A(t)$. If

$$
\begin{gathered}
\|A(t)\| \leqslant a, \\
\operatorname{Re} \lambda_{k}(t) \leqslant \lambda_{0}, \\
\|A(t)-A(s)\| \leqslant \delta|t-s|,
\end{gathered}
$$

then all solutions of the system admit the estimate

$$
\|x(t)\| \leqslant\left\|x\left(t_{0}\right)\right\| D_{\delta} e^{\left(\lambda_{0}+2 a \lambda_{\delta}\right)\left(t-t_{0}\right)} \quad\left(t \geqslant t_{0}\right)
$$

where

$$
\lambda_{\delta}=\left(C_{n} \cdot \delta / 4 a^{2}\right)^{1 /(n+1)}, \quad C_{n}=n(n+1) / 2,
$$

and $D_{\delta}$ depends only on $\delta$.

REMARKS. (i) In the trivial case $\delta=0$, i.e. $A(t)=$ const., $\lambda_{\delta}$ has to be replaced by an arbitrary $\varepsilon>0$ and $D_{\delta}$ by $D_{\varepsilon}$.

(ii) If $A(t)$ is differentiable, then (4) is equivalent to $\|\dot{A}(t)\| \leqslant \delta$.

(iii) (5) is true but trivial when $\lambda_{\delta} \geqslant 1$. So the method is of interest just for $\delta$ small, in other words, for systems (1) with "slowly changing" matrix $A(t)$.

We show that for $\delta$ small enough, the constant $C_{n}$ can be replaced by one close to

$$
C_{n}^{\prime}=2 n^{n} e^{-n} /(n-1) !
$$

Received by the editors November 17, 1982.

1980 Mathematics Subject Classification. Primary 34D05. 
Since by Stirling's Formula,

$$
C_{n}^{\prime}=2 n\left(n^{n} e^{-n} / n !\right)<\sqrt{2 n / \pi},
$$

we have $C_{n}^{\prime}<C_{n}$ for $n=1,2, \ldots$ and $C_{n}^{\prime}=o\left(C_{n}\right)$.

1. THEOREM 1. Let (2)-(4) hold. Then given $\varepsilon, 0<\varepsilon \leqslant(n+2)^{2} / 2$, there is $\delta(\varepsilon)>0$ such that for $\delta<\delta(\varepsilon)$ estimate (5) holds with

$$
\lambda_{\delta}=\left[\left(C_{n}^{\prime}+\varepsilon\right) \delta / 4 a^{2}\right]^{1 /(n+1)}, \quad C_{n}^{\prime}=2 n^{n} e^{-n} /(n-1) ! .
$$

The value of $\delta(\varepsilon)$ can be expressed explicitly:

$$
\delta(\varepsilon)=4 a^{2} \cdot \varepsilon^{n+1}\left[2 /(n+2)^{2}\right]^{n+2} .
$$

The trivial case $\delta=0$ is as in Remark (i).

To prove this theorem we need a number of preliminary steps.

2. The "frozen" equation. For simplicity we let $t_{0}=0$ in (5); the general case can be treated quite similarly-just replace $(0, t)$ with $\left(t_{0}, t_{0}+t\right)$.

Fix a value $t_{1}$ ("the point of freezing") and rewrite (1) as

$$
\dot{x}=A\left(t_{1}\right) x+\left[A(t)-A\left(t_{1}\right)\right] x .
$$

Then by the Variation of Constants Formula, we have for every solution $x(t)$ of (1):

$$
x(t)=e^{A\left(t_{1}\right) t} x(0)+\int_{0}^{t} e^{A\left(t_{1}\right)(t-s)}\left[A(s)-A\left(t_{1}\right)\right] x(s) d s .
$$

Notice that this is an identity in $t_{1}$. Therefore $t_{1}$ can be chosen arbitrarily, in particular being a function of $t$. A proper choice of $t_{1}$ will play the crucial role.

3. To estimate the norms in (9) we need the following well-known inequality (e.g. see [1] or [2]): If (2) and (3) hold, then

$$
\left\|e^{A\left(t_{1}\right) \tau}\right\| \leqslant p(2 a \tau) e^{\lambda_{0} \tau}
$$

where $p=p_{n-1}$ and

$$
p_{k}(z)=1+z / 1 !+\cdots+z^{k} / k !
$$

Let

$$
\|x(t)\|=\|x(0)\| e^{\left(\lambda_{0}+2 a \lambda\right) t} u(t)
$$

and $t_{1}=t-y / 2 a$, where $\lambda>0$ and $y$ will be chosen later. Then taking norms in (9) and using (4) and (10) we get

$$
u(t) \leqslant p(2 a t) e^{-2 a \lambda t}+\delta \int_{0}^{t}\left|t-s-\frac{y}{2 a}\right| p(2 a(t-s)) e^{-2 a \lambda(t-s)} u(s) d s .
$$

Now apply a particular case of the general Cone Theorem (e.g. see [3]).

Consider an integral inequality

$$
u(t) \leqslant f(t)+\int_{0}^{t} F(t, s) u(s) d s \quad(t \geqslant 0)
$$


where all functions are real valued, continuous and nonnegative. If $f(t)$ is bounded and

$$
\int_{0}^{t} F(t, s) d s \leqslant q<1 \quad \text { for all } t \geqslant 0,
$$

then $u(t)$ is bounded: $u(t) \leqslant \sup f(t) /(1-q)(t \geqslant 0)$.

In our case $f(t)=p(2 a t) e^{-2 a \lambda t}$ is clearly bounded. So if we manage to prove that

$$
I=\delta \int_{0}^{t}\left|t-s-\frac{y}{2 a}\right| \cdot p(2 a(t-s)) e^{-2 a \lambda(t-s)} d s \leqslant q<1
$$

for $\delta$ and $\lambda=\lambda_{\delta}$ as in Theorem 1, then $u(t)$ will be bounded and (5) will follow by (12). (The bound for $u$ depends on $\delta$; that is why $D_{\delta}$ appears in (5).)

4. Minimization of the integral. First transform I to a simpler form. Letting $2 a(t-s)=r$ and $\delta / 4 a^{2}=\gamma$, we have

$$
I=\gamma \int_{0}^{2 a t}|r-y| \cdot p(r) e^{-\lambda r} d r \leqslant \gamma \int_{0}^{\infty}|r-y| \cdot p(r) e^{-\lambda r} d r .
$$

Now choose $y$ to minimize the integral

$$
J=\int_{0}^{\infty}|r-y| \cdot p(r) e^{-\lambda r} d r=\int_{0}^{y}+\int_{y}^{\infty} .
$$

Setting $d J / d y$ equal to zero gives the equation in $y$ :

$$
\int_{0}^{y} p(r) e^{-\lambda r} d r=\int_{y}^{\infty} p(r) e^{-\lambda r} d r,
$$

which clearly has a unique positive root $y_{0}$. Then (13) yields

$$
J_{\min }=-\int_{0}^{y_{0}} r p(r) e^{-\lambda r} d r+\int_{y_{0}}^{\infty} r p(r) e^{-\lambda r} d r .
$$

The change of variables $z=\lambda y_{0}$ is convenient, and then the direct evaluation of the above integrals via the elementary formula

$$
\int P(x) e^{-\lambda x} d x=-e^{-\lambda x}\left[\frac{P(x)}{\lambda}+\frac{P^{\prime}(x)}{\lambda^{2}}+\cdots+\frac{P^{(m)}(x)}{\lambda^{m+1}}\right]+C,
$$

valid for every $m$ th degree polynomial $P(x)$, shows that (14) takes the form

$$
p_{\lambda}(z)=\frac{1}{2} e^{z} \text { or } 2 p_{\lambda}(z) e^{-z}=1
$$

where

$$
p_{\lambda}(z)=1+\frac{\Lambda_{n-2}}{\Lambda_{n-1}} \frac{z}{1 !}+\frac{\Lambda_{n-3}}{\Lambda_{n-1}} \frac{z^{2}}{2 !}+\cdots+\frac{1}{\Lambda_{n-1}} \frac{z^{n-1}}{(n-1) !}
$$

and $\Lambda_{k}=1+\lambda+\cdots+\lambda^{k}$, while

$$
J_{\min }=\frac{a_{1}(z)}{\lambda^{2}}+\frac{a_{2}(z)}{\lambda^{3}}+\cdots+\frac{a_{n}(z)}{\lambda^{n+1}},
$$

where (see (11)) $a_{k}(z)=k\left[2 p_{k}(z) e^{-z}-1\right]$.

Therefore the only task now is to prove

$$
\gamma J_{\min }=\gamma\left[a_{1}(z) / \lambda^{2}+\cdots+a_{n}(z) / \lambda^{n+1}\right]<1
$$

for $\gamma=\delta / 4 a^{2}$ and $\lambda=\lambda_{\delta}$ as in Theorem 1 . 
5. Recall that $z$ denotes the only positive root of (15) whose existence has already been established. Also notice that $p_{k}(z) e^{-z} \leqslant e^{z} e^{-z}=1$ and hence $a_{k}(z) \leqslant k$.

This estimate will suffice for $k \leqslant n-1$, but $a_{n}(z)$ has to be found more explicitly. We have

$$
a_{n}(z)=n\left[2 p_{n}(z) e^{-z}-1\right]=n\left[\frac{2 z^{n} e^{-z}}{n !}+2 p_{n-1}(z) e^{-z}-1\right] .
$$

Since the function $z^{n} e^{-z}(z \geqslant 0)$ takes on its maximum at $z=n$, we have

$$
n \cdot \frac{2 z^{n} e^{-z}}{n !} \leqslant \frac{2 n^{n} e^{-n}}{(n-1) !}=C_{n}^{\prime} .
$$

Next,

$$
\begin{aligned}
2 p_{n-1} e^{-z}-1 & =2 e^{-z}\left(p_{\lambda}+p_{n-1}-p_{\lambda}\right)-1 \\
& =2 e^{-z}\left(p_{n-1}-p_{\lambda}\right) \quad(\text { by }(15)) \\
& =\lambda \cdot 2 e^{-z}\left[\frac{\lambda^{n-2}}{\Lambda_{n-1}} \cdot \frac{z}{1 !}+\frac{\lambda^{n-3}+\lambda^{n-2}}{\Lambda_{n-1}} \cdot \frac{z^{2}}{2 !}\right. \\
& \left.\quad+\cdots+\frac{1+\cdots+\lambda^{n-2}}{\Lambda_{n-1}} \cdot \frac{z^{n-1}}{(n-1) !}\right] \\
& \leqslant \lambda \cdot 2 e^{-z}\left[\frac{z}{1 !}+\frac{z^{2}}{2 !}+\cdots+\frac{z^{n-1}}{(n-1) !}\right] \\
& \leqslant \lambda \cdot 2 e^{-z} p_{n-1}(z) \leqslant 2 \lambda .
\end{aligned}
$$

Finally, $a_{n}(z)=a_{n}^{*}(z)+\lambda a_{n}^{* *}(z)$, where

$$
a_{n}^{*}(z) \leqslant C_{n}^{\prime}, \quad a_{n}^{* *}(z) \leqslant 2 n,
$$

so

$$
\begin{aligned}
J_{\min } & =\frac{a_{1}}{\lambda^{2}}+\cdots+\frac{a_{n-2}}{\lambda^{n-1}}+\frac{a_{n-1}+a_{n}^{* *}}{\lambda^{n}}+\frac{a_{n}^{*}}{\lambda^{n+1}} \\
& \leqslant \frac{1}{\lambda^{2}}+\cdots+\frac{n-2}{\lambda^{n-1}}+\frac{(n-1)+2 n}{\lambda^{n}}+\frac{C_{n}^{\prime}}{\lambda^{n+1}} .
\end{aligned}
$$

Notice that

$$
C_{n}^{\prime} \leqslant \sqrt{2 n / \pi}<(n+4) / 2 \text { for } n \geqslant 1,
$$

hence the sum of the coefficients in the previous line is

$$
B=1+\cdots+(n-2)+(n-1)+2 n+C_{n}^{\prime}<(n+2)^{2} / 2 .
$$

6. Estimating roots of some polynomials. Consider an equation in $\lambda(\lambda>0)$ :

$$
Q_{\gamma}(\lambda) \equiv \gamma\left[b_{1} / \lambda^{2}+\cdots+b_{n} / \lambda^{n+1}\right]=1,
$$

or equivalently

$$
\lambda^{n+1}=\gamma\left(b_{1} \lambda^{n-1}+\cdots+b_{n}\right),
$$

where $b_{k}>0, k=1, \ldots, n$, and $\gamma>0$. Let $b_{1}+\cdots+b_{n}=B$. 
LEMMA. (i) (19) has a unique positive root $\lambda_{\gamma}$.

(ii) $\lambda_{\gamma}<\lambda$ if and only if $Q_{\gamma}(\lambda)<1$.

(iii) If $B \gamma<1$, then $\lambda_{\gamma}<(B \gamma)^{1 /(n+1)}$.

(iv) If $0<\varepsilon<B$ and

$$
\gamma<\gamma(\varepsilon)=\varepsilon^{n+1} / B^{n+2}
$$

then

$$
\lambda_{\gamma}<\left[\left(b_{n}+\varepsilon\right) \gamma\right]^{1 /(n+1)}
$$

Proof. (i) and (ii) are clear because $Q_{\gamma}(\lambda)$ strictly decreases from $\infty$ to 0 as $\lambda$ ranges from 0 to $\infty$. (iii) If $B \gamma<1$, then $Q_{\gamma}(1)=B \gamma<1$, and by (ii), $\lambda_{\gamma}<1$. Then (20) shows that $\lambda_{\gamma}^{n+1}=\gamma\left(b_{1} \lambda_{\gamma}^{n}+\cdots+b_{n}\right)<B \gamma$. (iv) Given (21) where $0<\varepsilon<B$, we have $B \gamma<(\varepsilon / B)^{n+1}<1$, and so by (iii), $\lambda_{\gamma}<(B \gamma)^{1 /(n+1)}<1$. But then

$$
b_{1} \lambda_{\gamma}^{n-1}+\cdots+b_{n-1} \lambda_{\gamma}<\left(b_{1}+\cdots+b_{n-1}\right) \lambda_{\gamma}<B \lambda_{\gamma}<B(B \gamma)^{1 /(n+1)}<\varepsilon .
$$

Now (20) implies (22).

7. Proof of Theorem 1. Look first at the equation (cf. (17))

$$
Q_{\gamma}(\lambda)=\gamma\left[\frac{1}{\lambda^{2}}+\cdots+\frac{(n-1)+2 n}{\lambda^{n}}+\frac{C_{n}^{\prime}}{\lambda^{n+1}}\right]
$$

in which, by $(18), B<(n+2)^{2} / 2$. Given $0<\varepsilon \leqslant(n+2)^{2} / 2$, let

$$
\left.\gamma(\varepsilon)=\varepsilon^{n+1}\left[2 /(n+2)^{2}\right]^{n+2}<\varepsilon^{n+1} / B^{n+2}\right)
$$

which is exactly (8). Now fix $0<\gamma_{0}<\gamma(\varepsilon)$ and set

$$
\lambda_{\delta} \equiv \lambda_{0}=\left[\left(C_{n}^{\prime}+\varepsilon\right) \gamma_{0}\right]^{1 /(n+1)},
$$

which is just (7). Let $z_{0}$ be the root of (15) with this fixed $\lambda_{0}$. Then all $a_{k}\left(z_{0}\right)$ become fixed, and by (17), $\gamma_{0} J_{\min } \leqslant Q_{\gamma_{0}}\left(\lambda_{0}\right)$.

Look at the equation $Q_{\gamma_{0}}(\lambda)=1$. By Lemma (iv), its root is

$$
\lambda_{\gamma_{0}}<\left[\left(C_{n}^{\prime}+\varepsilon\right) \gamma_{0}\right]^{1 /(n+1)}=\lambda_{0},
$$

and by Lemma (ii), $Q_{\gamma_{0}}\left(\lambda_{0}\right)<1$. So (16) holds, and the proof is completed.

\section{REFERENCES}

1. B. F. Bylov, D. M. Grobman, V. V. Nemyckī and R. E. Vinograd, The theory of Lyapunov exponents, “Nauka", Moscow, 1966, pp. 130-138. (Russian)

2. W. A. Coppel, Dichotomies in stability theory, Springer-Verlag, Berlin and New York, 1978, p. 4.

3. Yu. L. Daleckiĭ and M. G. Kreìn, Stability of solutions of differential equations in Banach space, Transl. Math. Mono., vol. 43, Amer. Math. Soc., Providence, R. I., 1974, p. 57.

Department of Mathematics, North Dakota State University, Fargo, North Dakota 58105 